\title{
MicroRNA-28 promotes the proliferation of non-small-cell lung cancer cells by targeting PTEN
}

\author{
FENGHE CUI*, QIAN ZHOU*, KUANG XIAO and HAIYUN QIAN \\ Department of Cardiothoracic Surgery, Jingzhou Central Hospital, The Second Clinical Medical College, \\ Yangtze University, Jingzhou, Hubei 434000, P.R. China
}

Received July 3, 2019; Accepted January 3, 2020

DOI: $10.3892 / \mathrm{mmr} .2020 .11033$

\begin{abstract}
Non-small-cell lung cancer (NSCLC) is the fundamental form of lung cancer and the leading cause of cancer-related mortality in humans. Numerous studies have identified a role for microRNAs (miRs) in cell proliferation, invasion and metastasis in numerous types of cancer, including lung cancer. In the present study, the functional roles and molecular mechanisms of miR-28 in NSCLC tumorigenesis were investigated. Reverse transcription-quantitative PCR (RT-qPCR) was used to measure miR-28 expression levels in NSCLC tumor tissues and cell lines. A dual-luciferase assay was performed to observe the direct interaction between miR-28 and PTEN in A549 cells. Furthermore, the effect of miR-28 on the mRNA and protein expression levels of PTEN was examined by RT-qPCR and western blotting, respectively. A Cell Counting kit-8 assay was performed to identify the relationship between the miR-28/PTEN axis and tumor cell proliferation using cells infected with lentivirus (LV)-anti-miR-28 or LV-anti-miR-28 + short hairpin RNA-PTEN. miR-28 expression was upregulated in NSCLC tumor tissues and cell lines compared with the control groups. PTEN was identified as the downstream gene of miR-28 in NSCLC and was negatively regulated by miR-28. In addition, miR-28 knockdown suppressed the proliferation of A549 and H292 cells. Cells infected with LV-anti-miR-28 + short hairpin RNA-PTEN promoted tumor cell proliferation in A549 and H292 cells compared with cells infected with LV-anti-miR-28. Taken together, the present study suggested that miR-28 might serve as the promoter in the development of NSCLC by
\end{abstract}

Correspondence to: Dr Haiyun Qian, Department of Cardiothoracic Surgery, Jingzhou Central Hospital, The Second Clinical Medical College, Yangtze University, 1 Nanhuan Road, Jingzhou, Hubei 434000, P.R. China

E-mail: qhyfl@163.com

${ }^{*}$ Contributed equally

Key words: non-small-cell lung cancer, microRNA-28, PTEN, proliferation targeting PTEN. Therefore, the miR-28/PTEN axis may serve as a potential diagnostic and therapeutic target for NSCLC.

\section{Introduction}

Lung cancer is a malignant carcinoma, and a leading cause of morbidity and mortality worldwide $(1,2)$. The disease can be divided into two main types, according to clinicopathological features, namely, small-cell lung cancer and non-small-cell lung cancer (NSCLC) (3). NSCLC accounts for $80-90 \%$ of all cases of lung cancer $(4,5)$. Although the diagnostic and clinical therapeutic targets of NSCLC have significantly improved in the last decade, the high recurrence rate and poor 5-year survival rate remain (6). Thus, the molecular mechanism underlying NSCLC tumorigenesis requires further investigation.

Previous studies have confirmed the involvement of microRNAs (miRNAs/miRs) in the development of NSCLC $(4,7)$. miRNAs are a series of short ( 22 nucleotides), single-stranded, non-coding RNAs, which are mainly transcribed from introns or exons of protein-coding genes (8). miRNAs are involved in the post-transcriptional regulation of gene expression in multicellular organisms; they regulate genes by binding to the 3'-untranslated regions (3'-UTRs) of target mRNAs, thus decreasing the level of oncoprotein translation $(5,9)$. Previous studies have reported that miRNA dysregulation occurs in numerous types of human carcinoma, and that miRNAs are involved in tumor cell proliferation, apoptosis and metastasis $(10,11)$. According to the functional roles of downstream genes, miRNAs can act as oncogenes or tumor suppressors in different types of human cancer, including NSCLC $(12,13)$. For example, aberrant miR-28 expression has been detected in gastric cancer (14), ovarian cancer (15), B-cell lymphoma (16), colorectal cancer (17), hepatocellular carcinoma (18) and renal cell carcinoma (19). However, the detailed molecular mechanism of miR-28 in NSCLC has not been fully elucidated.

The present study aimed to identify the expression levels, functional roles and molecular mechanisms of miR-28, and to investigate the target genes associated with tumor cell proliferation, in NSCLC progression. The present study suggested that miR-28 acted as a promoter in the process of NSCLC cell proliferation by targeting PTEN. Furthermore, it was proposed that the miR-28/PTEN axis may serve as a novel clinical therapeutic target for NSCLC. 


\section{Materials and methods}

NSCLC tissues and cell lines. Tumor tissues and matched adjacent non-tumor tissues $(<3 \mathrm{~cm}$ from the tumor margin) were harvested from 33 patients with NSCLC ( 21 men and 12 women; mean age, 48.9 years; age range, $35-72$ years) at Jingzhou Central Hospital between October 2015 and December 2018. Regarding TNM staging (20), 15 (45.45\%) patients were classified as TNM stage I or II, whereas $18(54.55 \%)$ patients were classified as TNM stage III or IV. Total tissues were stored at $-80^{\circ} \mathrm{C}$ for tissue repository establishment. The present study was approved by the Ethical Committee of the Jingzhou Central Hospital. Written informed consent was obtained from all patients.

The cell lines, including BEAS-2b, A549, H1650, H292, H1944 and H1299, were purchased from the Institute of Biochemistry and Cell Biology of the Chinese Academy of Sciences. All cell lines were cultured using RPMI-1640 medium supplemented with $10 \%$ fetal bovine serum (Gibco; Thermo Fisher Scientific, Inc.) and 1\% penicillin-streptomycin at $37^{\circ} \mathrm{C}$ with $5 \% \mathrm{CO}_{2}$.

Prediction of target genes of miR-28. TargetScan (version 3.1; www.targetscan.org/mamm_31) software was used to determine the candidate downstream target genes of miR-28.

Reverse transcription-quantitative PCR (RT-qPCR). The NSCLC tumor and matched adjacent normal tissues (100 mg) were ground in liquid nitrogen and the resulting cells underwent RNA extraction. The aforementioned cell lines were also used for RT-qPCR. Total RNA was extracted from the cells using TRIzol ${ }^{\circledR}$ reagent (Invitrogen; Thermo Fisher Scientific, Inc.), according to the manufacturer's protocol. Total RNA was reverse transcribed into cDNA using the iScript cDNA synthesis kit (cat. no. 1708890; Bio-Rad Laboratories, Inc.), according to the manufacturer's protocol. Subsequently, TaqMan ${ }^{\circledR}$ MicroRNA assays (Applied Biosystems; Thermo Fisher Scientific, Inc.) were used to detect the expression of miR-28 (miR-28-5p, forward, 5'-CGGATCCAGGCCCTT CAAGGACTTTCT-3' and reverse, 5'-CGAATTCACAGA GCTCCTGCTGTGTCA-3'), according to the manufacturer's protocol. miR-28 expression levels were normalized to the internal reference gene U6 (forward, 5'-GCTTCGGCAGCA CATATACTAAAAT-3' and reverse, 5'-CGCTTCACGAAT TTGCGTGTCAT-3'). SYBR Premix Ex Taq II (Takara Bio, Inc.) was used to determine PTEN mRNA expression levels. The following primer pairs were used for qPCR: PTEN forward, 5'-TGGATTCGACTTAGACTTGACCT-3' and reverse, 5'-GGTGGGTTATGGTCTTCAAAAGG-3'; and GAPDH forward, 5'-GACTCATGACCACAGTCCATGC-3' and reverse, 5'-AGAGGCAGGGATGATGTTCTG-3'. PTEN mRNA expression levels were normalized to the internal reference gene GADPH. PCR was performed using the 7500 Realtime PCR system (Applied Biosystems; Thermo Fisher Scientific, Inc.) with the following thermocycling conditions: $12 \mathrm{~min}$ at $96^{\circ} \mathrm{C}$; followed by 35 cycles of $15 \mathrm{sec}$ at $96^{\circ} \mathrm{C}$ and $1 \mathrm{~min}$ at $58^{\circ} \mathrm{C}$; and a final extension at $72^{\circ} \mathrm{C}$ for $5 \mathrm{~min}$. Relative expression levels were quantified using the $2^{-\Delta \Delta C q}$ method (21).

Cell transfection and infection. The miR-28 mimic (mimic-miR-28; GAGUUAUCUGACACUCGAGGAA) and its negative control (mimic-NC; UUCUCCGAACGU GUCACGU) were purchased from Shanghai GenePharma Co., Ltd. A549 cells $\left(2 \times 10^{5}\right.$ cells/well) were transfected with mimic-miR-28 or mimic-NC $(50 \mathrm{nmol} / \mathrm{l})$ to investigate the interaction between miR-28 and PTEN. Lentivirus (LV)-short hairpin RNA (sh)-PTEN (CCACAGCUAGAACUUAUCA AA), LV-anti-miR-28 (CUCAAUAGACUGUGAGCUCCU U), LV-anti-miR-28 + sh-PTEN or the LV-NC (UUCUCC GAACGUGUCACGU) were infected (MOI=3) into A549 and $\mathrm{H} 292$ cells ( $2 \times 10^{5}$ cells/well). The lentiviruses used in the present study were purchased from Shanghai GenePharma Co., Ltd. Mimic transfection was performed using Lipofectamine ${ }^{\circledR}$ 3000 (Invitrogen; Thermo Fisher Scientific, Inc.). Following incubation for $6 \mathrm{~h}$ at $37^{\circ} \mathrm{C}$, the cell culture medium was replaced with fresh medium. At $24 \mathrm{~h}$ post-transfection, cells were used for subsequent experiments.

Luciferase activity assay. The luciferase reporter plasmid pGL3 (Shanghai GenePharma Co., Ltd.) was used to perform the luciferase activity assay. Renilla luciferase was used as the normalization control. A549 cells $\left(5 \times 10^{5}\right.$ cells/well) were seeded into a 6-well plate. The sequences of the wild-type (WT) PTEN 3'-UTR (UCCCAAGUCCUUUGUAGCUCCUC) and the mutant (MUT) PTEN 3'-UTR (UCCCAAGUCCUUUGU UCGAGGAC) were obtained from Shanghai GenePharma Co., Ltd. and were cloned into the pGL3 vector. Subsequently, A549 cells were transfected with pGL3-WT-PTEN-3'-UTR or pGL3-WUT-PTEN-3'-UTR $(1 \mu \mathrm{g} / \mathrm{ml})$ using Lipofectamine ${ }^{\circledR}$ 2000 (Invitrogen; Thermo Fisher Scientific, Inc.) and incubated for $6 \mathrm{~h}$ at $37^{\circ} \mathrm{C}$. A549 cells were also transfected with mimic-miR-28 or mimic-NC (50 nmol/l) using Lipofectamine ${ }^{\circledR}$ 2000 (Invitrogen; Thermo Fisher Scientific, Inc.) and incubated for $6 \mathrm{~h}$ at $37^{\circ} \mathrm{C}$. Subsequently, the cell culture medium was replaced with fresh medium. After $48 \mathrm{~h}$, the Dual-Luciferase Reporter assay system (Promega Corporation) was used to measure the luciferase activity, according to the manufacturer's instructions.

Western blotting. Total protein was extracted from A549 and H292 cells using 1 ml RIPA buffer (Beyotime Institute of Biotechnology). Total protein was quantified using a bicinchoninic acid protein assay kit (Beyotime Institute of Biotechnology). Protein ( $25 \mu \mathrm{g}$ ) was separated by SDS-PAGE using $10 \%$ gels and transferred onto PVDF membranes. The membranes were blocked with 5\% non-fat milk at room temperature for $2 \mathrm{~h}$. Subsequently, the membranes were incubated with primary antibodies against PTEN (cat. no. 60300-1-Ig; 1:1,000; Proteintech Group, Inc.) and GAPDH (cat. no. 60004-1-Ig; 1:20,000; Proteintech Group, Inc.) overnight at $4^{\circ} \mathrm{C}$. The membranes were then washed with TBS- $0.5 \%$ Tween 20 and incubated with a horseradish peroxidase-conjugated secondary antibody (cat. no. ab205719; 1:20,000; Abcam) for $2 \mathrm{~h}$ at room temperature. Protein bands were visualized using an ECL kit (EMD Millipore) and were imaged using a Bioshine ChemiQ 4600 Mini Chemiluminescence Imaging system (Ouxiang). Protein expression was quantified using Image $\mathbf{J}$ software (version 1.49; National Institutes of Health).

Cell Counting Kit-8 (CCK-8) assay. The CCK-8 assay was used to determine the proliferation of A549 and H292 cells 

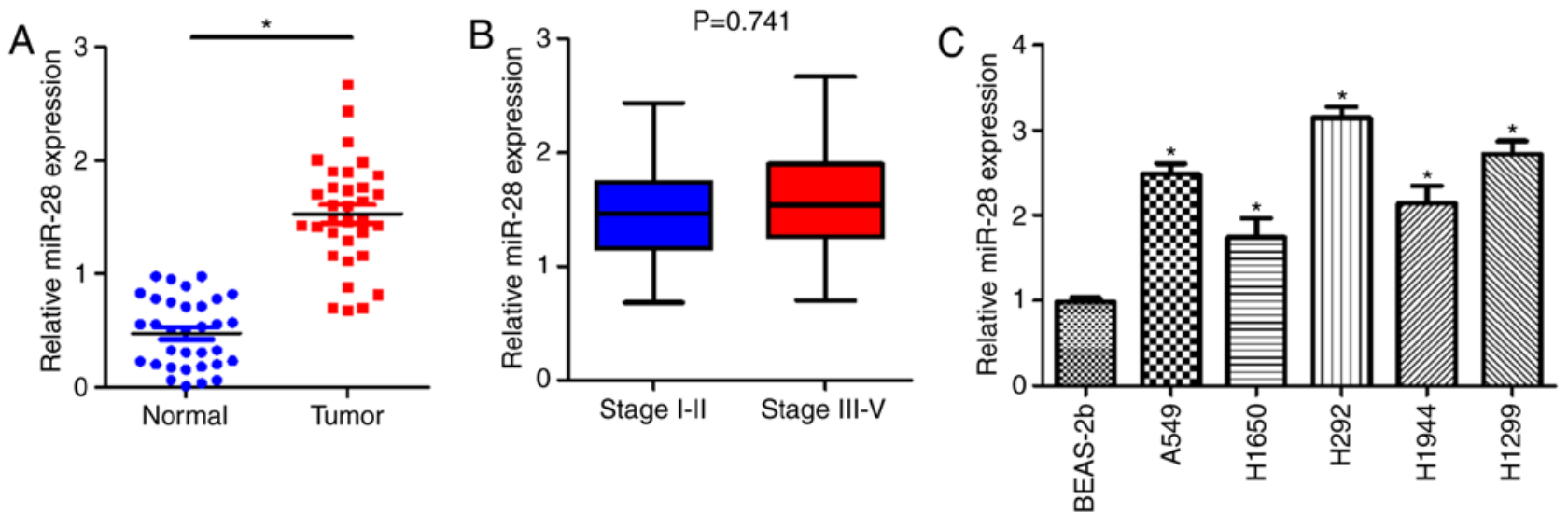

Figure 1. miR-28 expression is upregulated in NSCLC tumor tissues and cell lines. (A) Expression levels of miR-28 were measured in tumor tissues and paired adjacent non-tumor tissues of 33 patients with NSCLC. " $\mathrm{P}<0.05$. (B) Expression levels of miR-28 in NSCLC tumor tissues at different TNM stages. (C) Expression levels of miR-28 in a series of NSCLC cell lines, including A549, H1650, H292, H1944 and H1299. The BEAS-2b cell line represents normal human pulmonary epithelial cells and acted as the control group. ${ }^{*} \mathrm{P}<0.05 \mathrm{vs}$. the BEAS-2b group. All experiments were performed in triplicate. miR, microRNA; NSCLC, non-small-cell lung cancer.
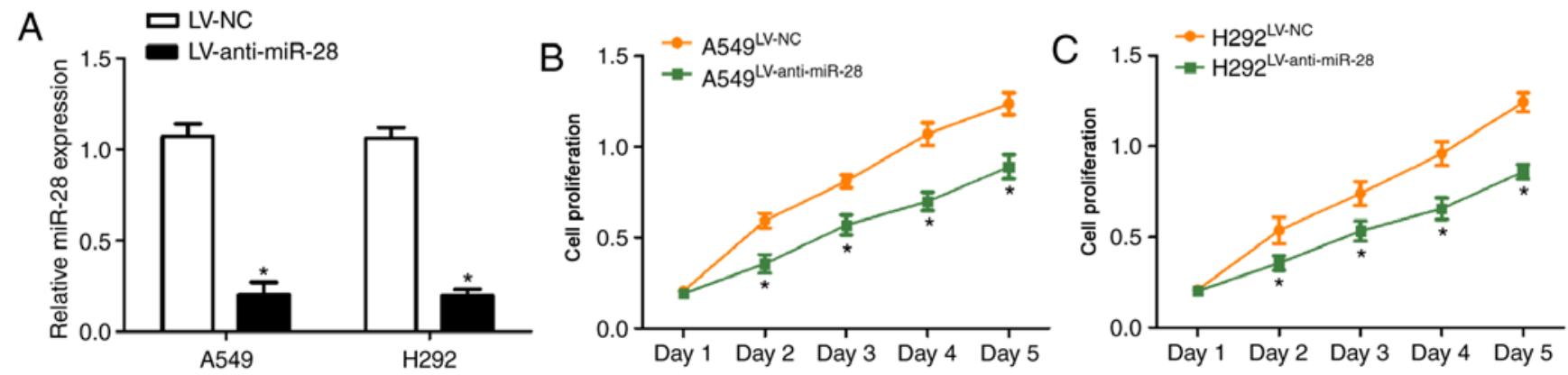

Figure 2. miR-28 knockdown reduces proliferation of A549 and H292 cells. (A) Expression levels of miR-28 in A549 and H292 cells infected with LV-anti-miR-28 or LV-NC. miR-28 expression levels were decreased following infection with LV-anti-miR-28. Cell proliferation was measured by a Cell Counting kit-8 assay in (B) A549 and (C) H292 cells infected with LV-anti-miR-28 or LV-NC, every 24 h for 5 days. All experiments were performed in triplicate. ${ }^{*} \mathrm{P}<0.05$. LV, lentivirus; miR, microRNA; NC, negative control.

(5x103 cells/well). The CCK-8 reagent (10 $\mu \mathrm{l}$; APExBIO Technology) was added to each well and subsequently, the cells were counted every $24 \mathrm{~h}$ for 5 days using a Fluoroskan Ascent microplate fluorometer (Thermo Fisher Scientific, Inc.). The optical density at a wavelength of $450 \mathrm{~nm}$ was measured. After 5 days, the growth curve was generated.

Statistical analysis. All experiments were performed in triplicate and repeated three times. The data are presented as the mean $\pm \mathrm{SD}$. A paired Student's t-test was used for the comparison of miR-28 expression between NSCLC tumor and matched adjacent normal tissues. One-way ANOVA followed by Tukey's post hoc test was used to measure the differences between quantitative variables. $\mathrm{P}<0.05$ was considered to indicate a statistically significant difference. GraphPad Prism (version 7.0; GraphPad Software, Inc.) and R studio (version 3.5.1; www.r-project.org) software were used to perform the statistical analyses.

\section{Results}

Upregulation of miR-28 expression in NSCLC tissues and cell lines. To investigate whether miR-28 was associated with
NSCLC development, RT-qPCR was performed to measure the expression levels of miR-28 in NSCLC tumor tissues and cell lines. miR-28 expression was significantly upregulated in NSCLC tissues compared with matched adjacent non-tumor tissues (Fig. 1A). There was no significant difference between the expression levels of miR-28 in early (1.497 in stage $\mathrm{I} / \mathrm{II}$ ) and late TNM stage tumors (1.553 in stage III/IV; P $>0.05$; Fig. 1B). The expression levels of miR-28 in A549, H1650, H292, H1944 and H1299 cell lines were significantly higher than in the BEAS-2b cell line (Fig. 1C), which is a normal lung bronchus epithelial cell line and acted as the control group. The results suggested that miR-28 was not only involved in the progression of NSCLC, but was also upregulated in NSCLC tumor tissues and cell lines.

miR-28 knockdown reduces proliferation of A549 and H292 cells. To determine the biological functions of miR-28 in the development of NSCLC, A549 and H292 cells were infected with LV-anti-miR-28. miR-28 expression levels were significantly decreased in both cell lines following infection with LV-anti-miR-28 compared with those infected with LV-NC (Fig. 2A), indicating that the infection was successful. The cell proliferation curves generated by performing the 

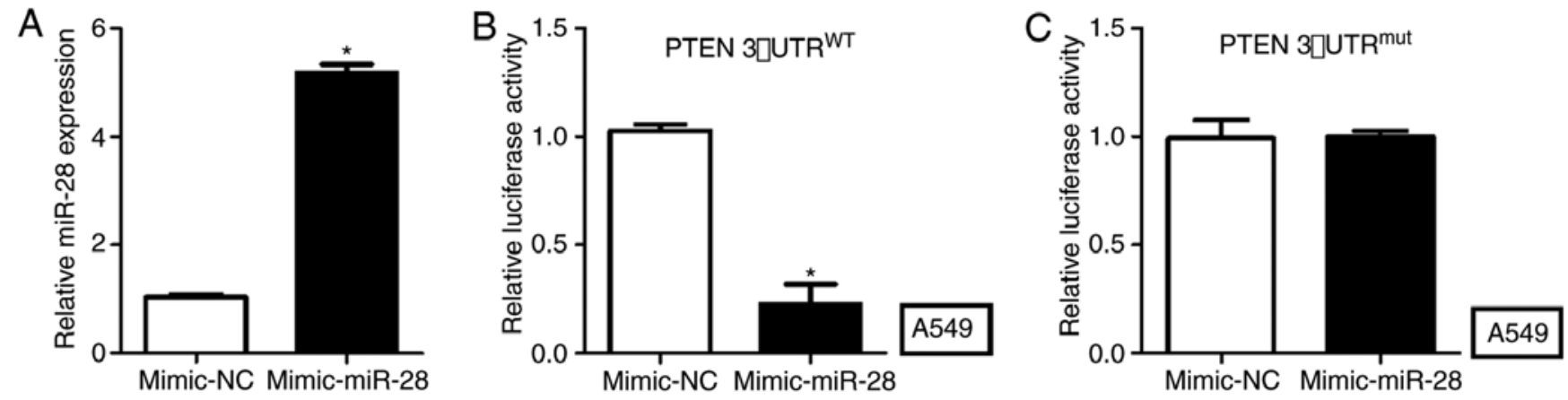

Figure 3. PTEN is the direct target gene of miR-28. (A) Expression levels of miR-28 in A549 cells transfected with mimic-miR-28 or mimic-NC. A dual-luciferase assay was performed to detect the relative luciferase activity in A549 cells co-transfected with mimic-miR-28 or mimic-NC and (B) WT-PTEN 3'-UTR or (C) MUT-PTEN 3'-UTR. "P<0.05 vs. mimic-NC. 3'-UTR, 3'-untranslated region; miR, microRNA; MUT, mutant; NC, negative control; WT, wild-type.
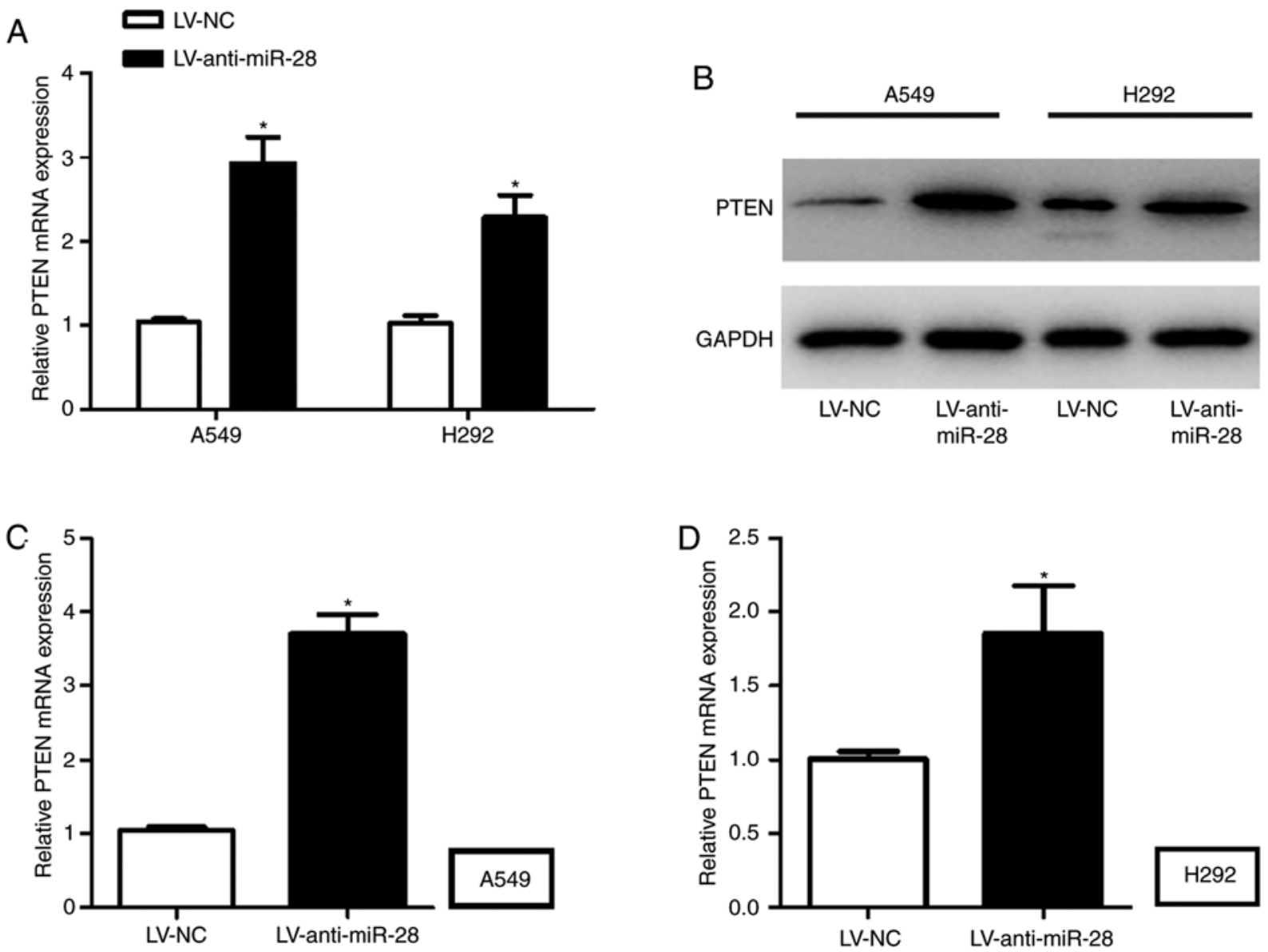

Figure 4. PTEN is negatively regulated by miR-28. (A) mRNA expression levels of PTEN in A549 and H292 cells infected with LV-anti-miR-28 or LV-NC. (B) Protein expression levels of PTEN in A459 and H292 cells infected with LV-anti-miR-28 determined by western blot analysis. Semi-quantification of PTEN protein expression in (C) A459 and (D) H292 cells infected with LV-anti-miR-28 or LV-NC. All experiments were performed in triplicate. "P<0.05. LV, lentivirus; miR, microRNA; NC, negative control.

CCK-8 assay suggested that miR-28 knockdown inhibited proliferation of A549 and H292 cells compared with the LV-NC group (Fig. 2B and C). The results indicated that miR-28 might act as a promoter in NSCLC progression.

PTEN is the direct target gene of $m i R-28$ and is negatively regulated by $m i R-28$. PTEN was predicted as the downstream gene of miR-28 using TargetScan (www.targetscan.org). To verify whether miR-28 directly targeted PTEN in NSCLC, a dual-luciferase activity assay was performed in A549 cells. miR-28 expression levels were significantly increased in A549 cells transfected with mimic-miR-28 compared with those transfected with mimic-NC (Fig. 3A). The luciferase activity in A549 cells co-transfected with WT-PTEN-3'-UTR and mimic-miR-28 was significantly decreased compared with the mimic-NC group (Fig. 3B). The luciferase activity of A549 cells transfected with MUT-PTEN-3'-UTR was not significantly altered between cells co-transfected with mimic-miR-28 or mimic-NC (Fig. 3C). 

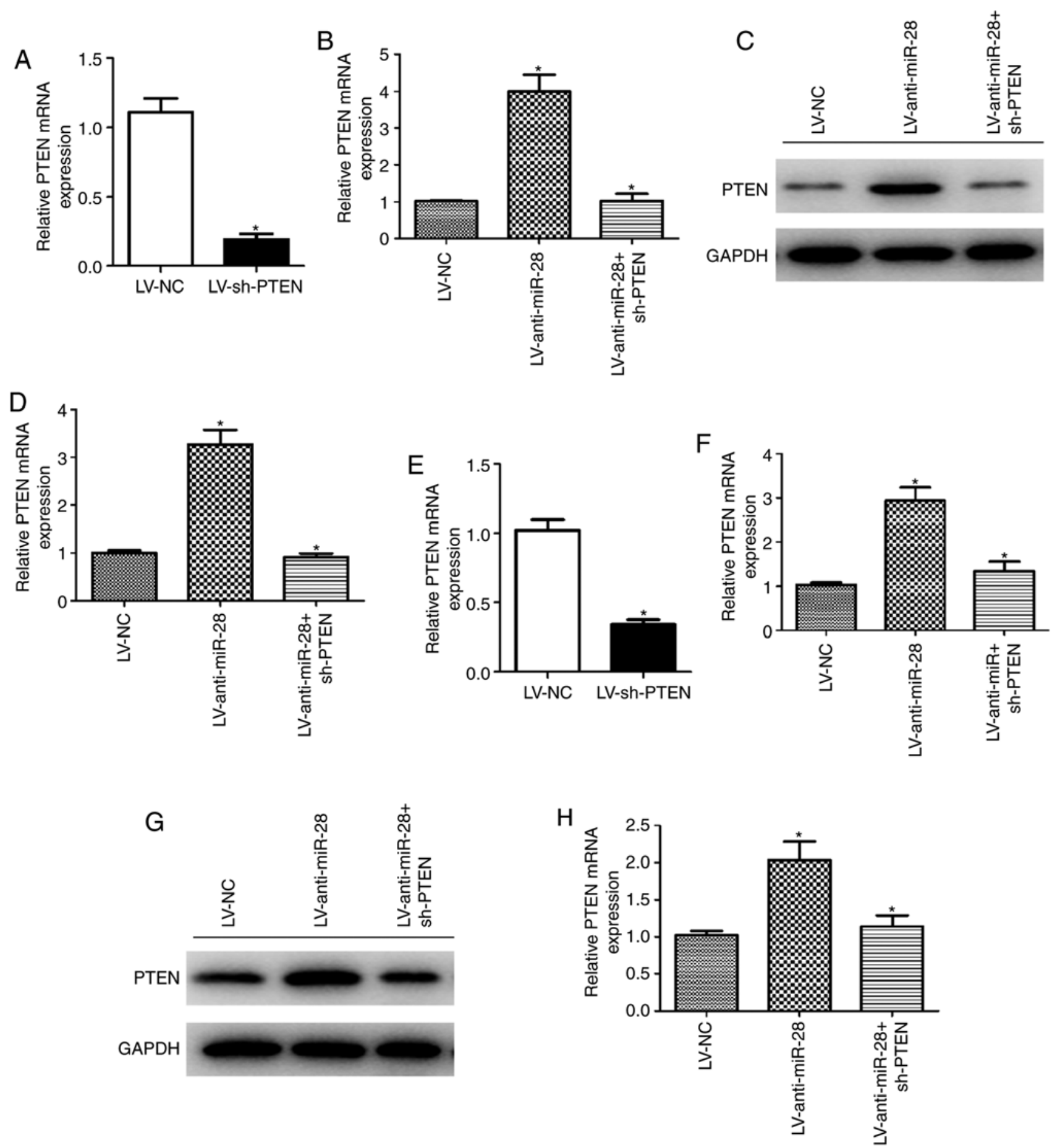

Figure 5. PTEN knockdown reverses the effect of miR-28 on PTEN expression in A549 and H292 cells. mRNA expression levels of PTEN in A549 cells infected with (A) LV-NC or LV-sh-PTEN, and (B) LV-anti-miR-28, LV-anti-miR-28 + sh-PTEN or LV-NC. Protein expression levels of PTEN in A549 cells infected with LV-anti-miR-28, LV-anti-miR-28 + sh-PTEN or LV-NC were (C) determined by western blot analysis and (D) semi-quantified. (E) mRNA expression levels of PTEN in $\mathrm{H} 292$ cells infected with (E) LV-NC or LV-sh-PTEN, and (F) LV-anti-miR-28, LV-anti-miR-28 + sh-PTEN or LV-NC. Protein expression levels of PTEN in H292 cells infected with LV-anti-miR-28, LV-anti-miR-28 + sh-PTEN or LV-NC (G) determined by western blot analysis and $(\mathrm{H})$ semi-quantified. All experiments were performed in triplicate. ${ }^{*} \mathrm{P}<0.05 \mathrm{vs.} \mathrm{LV-NC.} \mathrm{LV}$, lentivirus; miR, microRNA; NC, negative control; sh, short hairpin RNA.

To explore the direct effect of miR-28 on PTEN, RT-qPCR and western blotting were performed to measure the mRNA and protein expression levels of PTEN in A549 and H292 cells infected with LV-anti-miR-28. A549 and H292 cells infected with LV-anti-miR-28 displayed significantly higher PTEN mRNA and protein expression levels compared with A549 and H292 cells infected with LV-NC (Fig. 4A-D).
Collectively, these results suggested that PTEN may be the downstream gene of miR-28 in NSCLC; therefore, miR-28 knockdown promoted the expression of PTEN.

PTEN knockdown reverses the effect of anti-miR-28 on PTEN expression and cell proliferation in NSCLC. sh-PTEN was used to investigate whether PTEN affected the progression of 

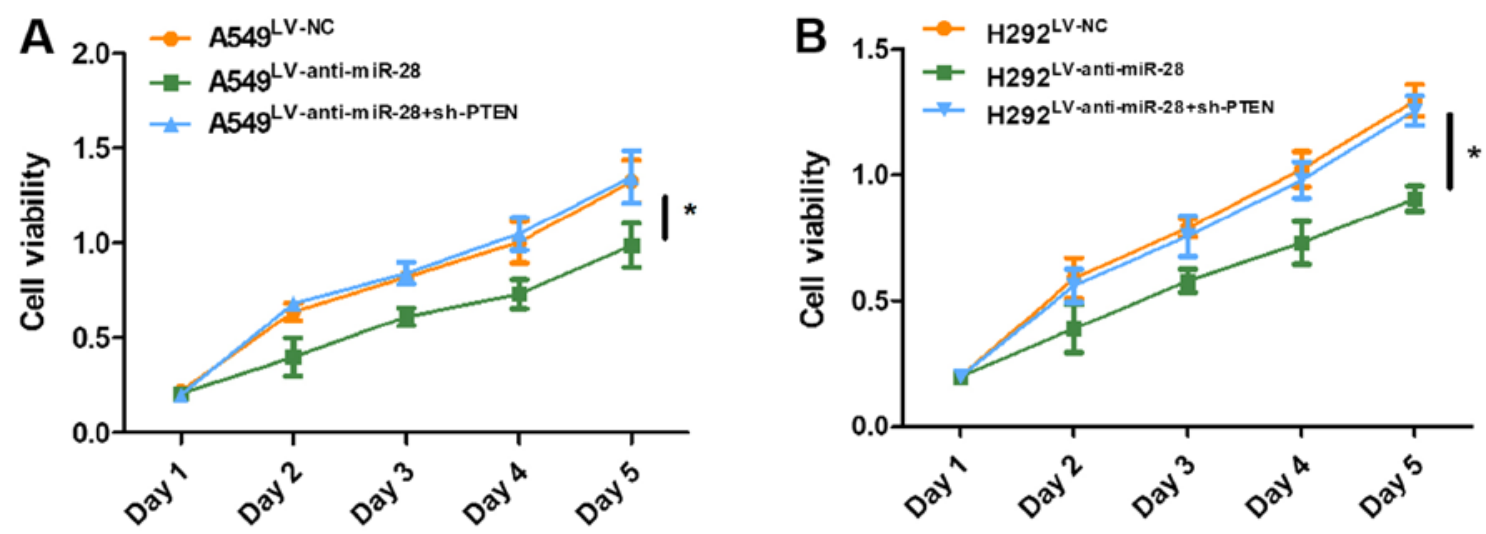

Figure 6. PTEN knockdown reverses the effect of anti-miR-28 on proliferation of A549 and H292 cells. Proliferation of (A) A549 and (B) H292 cells infected with LV-anti-miR-28, LV-anti-miR-28 + sh-PTEN or LV-NC; proliferation was detected every $24 \mathrm{~h}$ for 5 days. All experiments were performed in triplicate. "P<0.05. LV, lentivirus; miR, microRNA; NC, negative control; sh, short hairpin RNA.

NSCLC. PTEN expression levels were significantly decreased in A549 and H292 cells infected with LV-sh-PTEN compared with those infected with LV-NC (Fig. 5A and E). The mRNA and protein expression levels of PTEN in A549 and $\mathrm{H} 292$ cells co-infected with LV-anti-miR-28 + sh-PTEN were significantly lower compared with cells infected with LV-anti-miR-28 (Fig. 5B-D and F-H). The CCK-8 assay suggested that A549 and $\mathrm{H} 292$ cells co-infected with LV-anti-miR-28 + sh-PTEN displayed higher levels of cell proliferation compared with A549 and H292 cells infected with LV-anti-miR-28 (Fig. 6). The results indicated that PTEN knockdown reversed the inhibitory effect of LV-anti-miR-28 on cell proliferation. Furthermore, the results suggested that miR-28 might play a role as an oncogene in NSCLC by targeting PTEN.

\section{Discussion}

In the present study, the underlying functional roles of miR-28 in NSCLC tumorigenesis were investigated. miR-28 expression levels were significantly upregulated in NSCLC tumor tissues and cell lines compared with the matched adjacent non-tumor tissues and the control cell line, respectively. Additionally, PTEN was identified as the downstream gene of miR-28 during NSCLC development. miR-28 knockdown increased PTEN expression levels and reduced tumor cell proliferation in vitro. PTEN knockdown reduced the effect of miR-28 on NSCLC tumor cell proliferation. Overall, it could be suggested that miR-28 acted as a promoter in NSCLC by targeting PTEN. Therefore, the miR-28/PTEN axis may serve as a potential clinical target for NSCLC diagnosis, treatment and prognosis.

miR-28, which is located at chromosome $3 \mathrm{q} 28$, has two main subtypes, miR-28-3p and miR-28-5p (22). In previous studies, miR-28 and its two subtypes have been reported to be aberrantly expressed by certain pathological mechanisms. Zhou et al (23) reported that miR-28-3p expression levels were increased in the plasma of patients with pulmonary embolism. With regards to lymphocytic leukemia, miR-28-5p expression was significantly increased in patients compared with healthy controls (24). Platelet miR-28 expression was also identified as being upregulated in patients with myeloproliferative neoplasm (25). The aforementioned results indicated that
miR-28 upregulation commonly occurs in diseases, which is consistent with the findings of the present study.

miR-28 can act as an oncogene or a tumor suppressor in various types of malignant carcinoma. Schneider et al (16) reported that overexpression of miR-28inhibited cell proliferation in B-cell lymphoma. Xu et al (15) demonstrated that miR-28-5p induced the proliferation of ovarian cancer cells, as well as their migration and invasion, by targeting NEDD4-binding protein 1 . Wu et al (17) reported that miR-28-5p had a suppressive effect on colorectal cancer progression by interacting with the downstream gene structure specific recognition protein 1 . However, another similar study suggested that miR-28-3p acted as a tumor promoter in colorectal cancer cell migration and invasion (22). Therefore, the aforementioned studies illustrate that the effects of miR-28 are not always identical in different types of cancer and that the different subtypes of miR-28 may have opposite functions in the same cancer. The present study suggested that miR-28 served as an oncogene in NSCLC cell proliferation, which is supported by Wang et al (26) who reported that miR-28 is one of the potential oncogenes in lung cancer.

PTEN, located at chromosome 10q23.31, has been identified as a tumor suppressor gene via the PI3K/AKT pathway in a number of different forms of cancer (27), including renal cancer (28), gastric cancer (29), endometrial cancer (30), breast cancer (31) and malignant melanoma (32). Previously, emerging miRNAs have been identified as promoters of tumor cell growth, metastasis or apoptosis by targeting PTEN, including miR-1297 (33), miR-200 (34), miR-130a (35), miR-26a (36) and miR-17 (37). In the present study, PTEN was also identified as the target gene of miR-28. Additionally, the oncogenic role of miR-28 in NSCLC proposed in the present study was the same as that of miRNAs reported previously (33-37). These results suggested that miR-28 directly targeted PTEN and may promote tumorigenesis in NSCLC.

A previous study investigated the interaction of the miR-28/PTEN axis in carcinoma. Li et al (14) reported that miR-28 acts as an oncogene in gastric cancer growth and invasion by targeting PTEN, via the PI3K/AKT signaling pathway, which strengthened the present findings. Moreover, the function of the miR-28/PTEN axis in other types of human cancer requires further investigation and could identify additional biomarkers for cancer research. 
In conclusion, the present study suggested that miR-28 was upregulated in NSCLC tumor tissues and cell lines. Moreover, miR-28 promoted NSCLC cell proliferation by targeting PTEN, which serves as a suppressor of pathogenesis in numerous diseases (27). The present study identified a potential biomarker for the clinical diagnosis, treatment and prognosis of NSCLC. Further studies investigating whether there are other target genes of miR-28 in NSCLC and whether the miR-28/PTEN axis functions in other types of cancer are required.

\section{Acknowledgements}

Not applicable.

\section{Funding}

No funding was received.

\section{Availability of data and materials}

The datasets used and/or analyzed during the current study are available from the corresponding author on reasonable request.

\section{Authors' contributions}

HQ conceived and designed the present study. FC and QZ performed the experiments and analyzed the data. FC, QZ and KX helped to design the study and interpreted the data. All authors read and approved the final manuscript.

\section{Ethics approval and consent to participate}

The present study was approved by the Ethical Committee of the Jingzhou Central Hospital. Written informed consent was obtained from all patients.

\section{Patient consent for publication}

Not applicable.

\section{Competing interests}

The authors declare that they have no competing interests.

\section{References}

1. Castro D, Moreira M, Gouveia AM, Pozza DH and De Mello RA: MicroRNAs in lung cancer. Oncotarget 8: 81679-81685, 2017.

2. Wu KL, Tsai YM, Lien CT, Kuo PL and Hung AJ: The roles of MicroRNA in lung cancer. Int J Mol Sci 20: E1611, 2019.

3. Herbst RS, Heymach JV and Lippman SM: Lung cancer. N Engl J Med 359: 1367-1380, 2008.

4. Vannini I, Fanini F and Fabbri M: MicroRNAs as lung cancer biomarkers and key players in lung carcinogenesis. Clin Biochem 46: 918-925, 2013.

5. Zhang WC, Liu J, Xu X and Wang G: The role of microRNAs in lung cancer progression. Med Oncol 30: 675, 2013.

6. Angulo M, Lecuona E and Sznajder JI: Role of MicroRNAs in lung disease. Arch Bronconeumol (Spanish) 48: 325-330, 2012

7. Del Vescovo V, Grasso M, Barbareschi $M$ and Denti MA: MicroRNAs as lung cancer biomarkers. World J Clin Oncol 5 : 604-620, 2014
8. Alipoor SD, Adcock IM, Garssen J, Mortaz E, Varahram M, Mirsaeidi M and Velayati A: The roles of miRNAs as potential biomarkers in lung diseases. Eur J Pharmacol 791: 395-404, 2016.

9. Guz M, Rivero-Muller A, Okon E, Stenzel-Bembenek A Polberg K, Slomka M and Stepulak A: MicroRNAs-role in lung cancer. Dis Markers 2014: 218169, 2014.

10. Kang SM and Lee HJ: MicroRNAs in human lung cancer. Exp Biol Med (Maywood) 239: 1505-1513, 2014.

11. Wu SG, Chang TH, Liu YN and Shih JY: MicroRNA in lung cancer metastasis. Cancers (Basl) 11: E265, 2019.

12. Markou A, Sourvinou I, Vorkas PA, Yousef GM and Lianidou E: Clinical evaluation of microRNA expression profiling in non small cell lung cancer. Lung Cancer 81: 388-396, 2013.

13. Shen J, Liu Z, Todd NW, Zhang H, Liao J, Yu L, Guarnera MA, Li R, Cai L, Zhan M and Jiang F: Diagnosis of lung cancer in individuals with solitary pulmonary nodules by plasma microRNA biomarkers. BMC Cancer 11: 374, 2011.

14. Li L, Zhu X, Shou T, Yang L, Cheng X, Wang J, Deng L and Zheng Y: MicroRNA-28 promotes cell proliferation and invasion in gastric cancer via the PTEN/PI3K/AKT signalling pathway. Mol Med Rep 17: 4003-4010, 2018.

15. Xu J, Jiang N, Shi H, Zhao S, Yao S and Shen H: miR-28-5p promotes the development and progression of ovarian cancer through inhibition of N4BP1. Int J Oncol Mar 16, 2017 (Epub ahead of print).

16. Schneider C, Setty M, Holmes AB, Maute RL, Leslie CS, Mussolin L, Rosolen A, Dalla-Favera R and Basso K: MicroRNA 28 controls cell proliferation and is down-regulated in B-cell lymphomas. Proc Natl Acad Sci USA 111: 8185-8190, 2014.

17. Wu W, He K, Guo Q, Chen J, Zhang M, Huang K, Yang D, Wu L, Deng Y, Luo X, et al: SSRP1 promotes colorectal cancer progression and is negatively regulated by miR-28-5p. J Cell Mol Med 23: 3118-3129, 2019

18. Zhou SL, Hu ZQ, Zhou ZJ, Dai Z, Wang Z, Cao Y, Fan J, Huang XW and Zhou J: miR-28-5p-IL-34-macrophage feedback loop modulates hepatocellular carcinoma metastasis. Hepatology 63: 1560-1575, 2016.

19. Wang C, Wu C, Yang Q, Ding M, Zhong J, Zhang CY, Ge J, Wang $J$ and Zhang C: miR-28-5p acts as a tumor suppressor in renal cell carcinoma for multiple antitumor effects by targeting RAP1B. Oncotarget 7: 73888-73902, 2016.

20. Nicholson AG, Chansky K, Crowley J, Beyruti R, Kubota K, Turrisi A, Eberhardt WE and van Meerbeeck J; Staging and Prognostic Factors Committee, Advisory Boards, and Participating Institutions; Staging and Prognostic Factors Committee Advisory Boards and Participating Institutions: The international association for the study of lung cancer lung cancer staging project: Proposals for the revision of the clinical and pathologic staging of small cell lung cancer in the forthcoming eighth edition of the TNM classification for lung cancer. J Thoracic Oncol 11: 300-311, 2016.

21. Livak KJ and Schmittgen TD: Analysis of relative gene expression data using real-time quantitative PCR and the 2(-Delta Delta C(T)) method. Methods 25: 402-408, 2001.

22. Almeida MI, Nicoloso MS, Zeng L, Ivan C, Spizzo R, Gafà R, Xiao L, Zhang X, Vannini I, Fanini F, et al: Strand-specific miR-28-5p and miR-28-3p have distinct effects in colorectal cancer cells. Gastroenterology 142: 886-896.e9, 2012.

23. Zhou X, Wen W, Shan X, Qian J, Li H, Jiang T, Wang W, Cheng W, Wang F, Qi L, et al: MiR-28-3p as a potential plasma marker in diagnosis of pulmonary embolism. Thromb Res 138: 91-95, 2016.

24. Yang YQ, Tian T, Zhu HY, Liang JH, Wu W, Wu JZ, Xia Y, Wang L, Fan L, Li JY and Xu W: NDRG2 mRNA levels and miR-28-5p and miR-650 activity in chronic lymphocytic leukemia. BMC Cancer 18: 1009, 2018.

25. Girardot M, Pecquet C, Boukour S, Knoops L, Ferrant A, Vainchenker W, Giraudier S and Constantinescu SN: miR-28 is a thrombopoietin receptor targeting microRNA detected in a fraction of myeloproliferative neoplasm patient platelets. Blood 116: 437-445, 2010.

26. Wang QZ, Xu W, Habib N and Xu R: Potential uses of microRNA in lung cancer diagnosis, prognosis, and therapy. Curr Cancer Drug Targets 9: 572-594, 2009.

27. Hopkins BD and Parsons RE: Molecular pathways: Intercellular PTEN and the potential of PTEN restoration therapy. Clin Cancer Res 20: 5379-5383, 2014.

28. Zaman MS, Thamminana S, Shahryari V, Chiyomaru T, Deng G, Saini S, Majid S, Fukuhara S, Chang I, Arora S, et al: Inhibition of PTEN gene expression by oncogenic miR-23b-3p in renal cancer. PLoS One 7: e50203, 2012. 
29. Yang Z, Yuan XG, Chen J, Luo SW, Luo ZJ and Lu NH: Reduced expression of PTEN and increased PTEN phosphorylation at residue Ser380 in gastric cancer tissues: A novel mechanism of PTEN inactivation. Clin Res Hepatol Gastroenterol 37: 72-79, 2013.

30. Yoneyama K, Ishibashi O, Kawase R, Kurose K and Takeshita T: miR-200a, miR-200b and miR-429 are onco-miRs that target the PTEN gene in endometrioid endometrial carcinoma. Anticancer Res 35: 1401-1410, 2015.

31. Zhang WL and Zhang JH: miR-181c promotes proliferation via suppressing PTEN expression in inflammatory breast cancer. Int J Oncol 46: 2011-2020, 2015.

32. Zhou XP, Gimm O, Hampel H, Niemann T, Walker MJ and Eng C: Epigenetic PTEN silencing in malignant melanomas without PTEN mutation. Am J Pathol 157: 1123-1128, 2000.
33. Li X, Wang HL, Peng X, Zhou HF and Wang X: miR-1297 mediates PTEN expression and contributes to cell progression in LSCC. Biochem Biophys Res Commun 427: 254-260, 2012.

34. Wu Q, Lu RL, Li JX and Rong LJ: MiR-200a and miR-200b target PTEN to regulate the endometrial cancer cell growth in vitro. Asian Pac J Trop Med 10: 498-502, 2017.

35. Wei H, Cui R, Bahr J, Zanesi N, Luo Z, Meng W, Liang G and Croce CM: miR-130a deregulates PTEN and stimulates tumor growth. Cancer Res 77: 6168-6178, 2017.

36. Li B and Sun H: MiR-26a promotes neurite outgrowth by repressing PTEN expression. Mol Med Rep 8: 676-680, 2013.

37. Gao Y, Luo LH, Li S and Yang C: miR-17 inhibitor suppressed osteosarcoma tumor growth and metastasis via increasing PTEN expression. Biochem Biophys Res Commun 444: 230-234, 2014. 